\title{
Comparison of copper and tungsten carbide calibration spheres
}

\author{
Kenneth G. Foote \\ Institute of Marine Research, 5011 Bergen, Norway \\ David N. MacLennan \\ Marine Laboratory, Aberdeen, Scotland
}

(Received 1 April 1983; accepted for publication 2 November 1983)

\begin{abstract}
The properties of calibration spheres made from electrical-grade copper, denoted $\mathrm{Cu}$, and tungsten carbide with $6 \%$ cobalt binder, denoted WC, are examined theoretically. Effects of variations in material, medium, and equipment parameters are determined for spheres intended to calibrate $38-\mathrm{kHz}$ echo sounders when a target strength of order $-41.5 \mathrm{~dB}$ is required. The derived 30.4-mm-diam $\mathrm{Cu}$ sphere and selected 38.1-mm-diam WC ball bearing show different dependences on the varied parameters. Changes in the material and equipment parameters within wide ranges will not upset a precision calibration with expected \pm 0.1 -dB accuracy. Adjustment of the calibration value of backscattering cross section with changing temperature is generally necessary for elastic spheres. This is the case for the present WC sphere, but not for the Cu sphere by virtue of its optimization with respect to temperature. Greater hardness is found to be insufficient for choosing between the two; the particular application must be considered. Pragmatic criteria for sphere selection are discussed.
\end{abstract}

PACS numbers: 43.85. Vb, 43.20.Fn, 43.30.Dr

\section{INTRODUCTION}

Interest in the use of solid metal spheres as calibration or reference targets has increased significantly since their dismissal for presumably large-system calibrations by Wallace et al. ${ }^{1}$ Dragonette et al. ${ }^{2}$ have used tungsten carbide spheres for gauging backscattering measurements in the laboratory. Similar spheres have been examined in Scotland for possible application to echo sounder calibrations. ${ }^{3}$ Copper has also received much attention. ${ }^{3-6}$ Spheres machined from electrical-grade copper are being used to calibrate fisheries echo sounders in Norway and elsewhere. ${ }^{7}$ There have also been applications of solid metal spheres in nonlinear acoustics. ${ }^{8,9}$

Purely theoretical considerations have favored the socalled "group II" materials of Anson and Chivers' classification. ${ }^{10}$ This is somewhat misleading, for while they are generally hard and dense, they are also among the most expensive and hazardous, not to mention least machinable of materials. ${ }^{11,12}$ Some examples are beryllium, boron, sintered diamond, iridium, niobium, osmium, platinum, rhenium, ruthenium, and tungsten carbide. ${ }^{13}$ "Group III" materials, in contrast, while less hard and possessing more complicated radiation functions and backscattering cross sections than the group II materials, are generally much easier to fabricate. Copper belongs to this class, as do aluminum, nickel, monel metal, stainless steel, and zinc. ${ }^{13}$

The present study deals with pragmatic considerations in the choice of material for fabrication into spheres designed for use in hydroacoustic calibrations. The object materials, copper and tungsten carbide, are convenient both for their immediate and potential applications and for their representation of acoustically dissimilar materials according to Anson and Chivers' admittedly somewhat arbitrary classification. ${ }^{10}$ Since both materials have been used successfully for precision calibration, as has fused silica, ${ }^{14}$ which is also a group III material like copper, secondary aims of the study are to emphasize the futility of selecting materials solely on the basis of their hardness and to discourage misuse of the rigid-sphere approximation in the calculation of scattering properties.

\section{SPHERE SELECTION}

Selection of a sphere for calibrating any given acoustic instrument generally involves selection of the material and determination or selection of the diameter. This is now discussed both for copper and for tungsten carbide. As a practical example, the derived spheres are to be suitable for calibrating the $38-\mathrm{kHz}$ echo sounders commonly used in fish stock estimation. The calibrations are to be performed at short ranges, but in the farfield of the transducer. Furthermore, the target strength is to be roughly equal to that of a ping-pong ball; namely, about $-41.5 \mathrm{~dB} .{ }^{15}$

\section{A. Copper}

This noble metal has many virtues. ${ }^{4,7}$ It is cheap, corrosion resistant, easy to machine, readily available in a high state of purity, and hard when cold-worked and unannealed. ${ }^{16}$

With respect to the elasticity, which with the density determines the scattering response for a given immersion medium, the elemental composition of commercial electrical-grade copper is important. The effect of trace element content should be negligible. ${ }^{17-20}$ The two commercial electrical-grade forms of copper, the oxygen-free (OF) and the electrolytic tough-pitch (ETP), may be expected to have very similar elastic properties. ${ }^{21}$

Another property of copper which is advantageous to its elasticity, hence choice, is its cubic structure. Texturing is 
unlikely to influence this, which may explain the evident constancy of the rigidity modulus of commercial electricalgrade coppers. ${ }^{18,22-27}$ This appears to be very near $47 \mathrm{GPa}$, which corresponds to a Poisson's ratio of 0.350 , assuming a Lamé parameter $\lambda$ of $110 \mathrm{GPa}$, or a density of $8950 \mathrm{~kg} / \mathrm{m}^{3}$ and longitudinal sound speed of $4770 \mathrm{~m} / \mathrm{s}$.

The constancy in elasticity of OF and ETP coppers allows the acoustic characteristics of such spheres to be calculated reliably. The fact of copper being a group III material and having a frequency-sensitive backscattering cross section and radiation force function is thus no drawback to its choice. ${ }^{13}$ Indeed, this feature may be exploited to obtain high, or low, values of backscattering cross section or radiation force function for a sphere size which could not be achieved with the harder, more acoustically constant group II materials.

The diameter of the copper sphere is determined in accordance with the optimization scheme developed in Ref. 4. The backscattering cross section is to vary as little as possible over the temperature range from $0^{\circ}$ to $30^{\circ} \mathrm{C}$ in sea water of salinity $35 \mathrm{ppt}$. Given that the target strength must be about $-41.5 \mathrm{~dB}$, the optimal diameter is found to be $30.40 \mathrm{~mm}$. Machining a sphere of this size can be accomplished quite easily with a tolerance of $0.1 \%$, which earlier investigations have shown to be adequate. ${ }^{4,7}$

\section{B. Tungsten carbide}

Tungsten carbide with $6 \%$ cobalt added as a binder was chosen as a promising material. It is extremely hard-tungsten carbide is 9.5 on the Mohs hardness scale-and it is commercially available in the form of precision ball bearings. Only discrete sizes can be procured easily, but others can be obtained through the usual sintering process. Off-theshelf ball bearings are available in convenient sizes for the purpose of calibrating the acoustic equipment used in fisheries research, where frequencies are typically in the range from 30 to $150 \mathrm{kHz}$.

Tungsten carbide is a group II material. The frequency dependence of its form function follows that of the ideally hard sphere up to a wavenumber-radius product of about six. ${ }^{13}$

The diameter of the sphere is chosen from the standard range of ball bearing sizes to obtain the target strength nearest to $-41.5 \mathrm{~dB}$. The selected diameter is thus $38.1 \mathrm{~mm}$, i.e., 1.5 in., and the diameter tolerance is better than $0.01 \%$.

\section{METHOD}

The objects of the comparison are a 30.40-mm-diam sphere made from electrical-grade copper, denoted $\mathrm{Cu} 30.4$, and a 38.1-mm-diam sphere formed of tungsten carbide with $6 \%$ cobalt binder, denoted WC 38.1. Since these are intended for use in the calibration of $38-\mathrm{kHz}$ echo sounders, the operationally defined backscattering cross section $\sigma$ is the natural quantity for comparison. ${ }^{4}$ This is related to the incident signal spectrum $S$, the backscattering form function $F$, and the frequency response function $H$ by the well-known formula:

$$
\sigma=4 \pi \int_{0}^{\infty}|S F H|^{2} d \omega / \int_{0}^{\infty}|S H|^{2} d \omega
$$

where $\omega$ is the frequency. $S, F$, and $H$ are normally explicit functions of $\omega$.

In the computation below, $S$ is the spectrum of a pulsed sinusoid, and $H$ is the receiver response function of the Sim$\mathrm{rad}$ EK-400/38 echo sounder. ${ }^{4} F$ depends on the characteristics of both the sphere and the medium, in addition to the frequency. ${ }^{28}$ For the chosen homogeneous elastic materials, the relevant properties of the sphere are the radins $a$, the density $\rho_{1}$, the longitudinal sound speed $c_{1}$, and the transverse sound speed $c_{2}$. The medium properties are expressed through the density $\rho$ and the sound speed $c$. The nominal values of these quantities are given in Table $I$.

Variation of these parameters is the basis of the comparison, for discovery of an instability or other severe dependence of $\sigma$ would suggest that the sphere is unsuitable for use in precision calibration. In order to render the comparison as realistic as possible, the ranges of variation are chosen in accordance with the following principles.

Ideally, the material parameters would be varied over percentage ranges corresponding to the larger of the observed parameter ranges of the two materials. However, knowledge of the particular tungsten carbide in WC 38.1 is extremely limited, and generic tungsten carbides span too wide a range of densities and elasticities to be meaningful for the intended comparisons. ${ }^{29,30}$ More is known about variations in copper, and the parameter ranges are therefore derived from the literature on high-purity polycrystalline coppers. ${ }^{6}$ These are shown in Table II.

The medium parameters $\rho$ and $c$ both depend on the temperature $\tau$ and the salinity. The intended application is marine, so the salinity is chosen to be $35 \mathrm{ppt}$. Temperature variations from $0^{\circ}$ to $30^{\circ} \mathrm{C}$ are considered, as in earlier studies. ${ }^{4,7}$ The medium density and the sound speed are determined by the hydrographic parameters in the usual manner. ${ }^{31,32}$

Since the echo sounder may have to be calibrated in an untuned condition and might be used with different pulse durations, these parameters are varied over the ranges shown in Table II. The variation of the sphere diameter is redundant insofar as the scattering is a function of the wavenumber-radius product. Thus the same proportional change in frequency or diameter should produce the same change in form function. The examined $0.20-\mathrm{mm}$ range in diameter

TABLE I. Parameters and their nominal values for the two spheres, immersion medium, and equipment.

\begin{tabular}{|c|c|c|c|c|c|}
\hline Type & Name & Symbol & Units & $\begin{array}{rr} & \text { Spl } \\
\text { Cu } 30.4\end{array}$ & WC 38.1 \\
\hline Sphere & Diameter & $2 a$ & $\mathrm{~mm}$ & 30.4 & 38.1 \\
\hline Sphere & Density & $\rho_{1}$ & $\mathrm{~g} / \mathrm{cm}^{3}$ & 8.947 & 14.900 \\
\hline Sphere & $\begin{array}{l}\text { Longitudinal } \\
\text { sound speed }\end{array}$ & $c_{1}$ & $\mathrm{~km} / \mathrm{s}$ & 4.760 & 6.864 \\
\hline Sphere & $\begin{array}{l}\text { Transverse } \\
\text { sound speed }\end{array}$ & $c_{2}$ & $\mathbf{k m} / \mathbf{s}$ & 2.2885 & 4.1612 \\
\hline Medium & Density & $\rho$ & $\mathrm{g} / \mathrm{cm}^{3}$ & \multicolumn{2}{|c|}{1.027} \\
\hline Medium & Sound speed & $c$ & $\mathrm{~km} / \mathrm{s}$ & \multicolumn{2}{|c|}{1.490} \\
\hline Equipment & $\begin{array}{l}\text { Center } \\
\text { frequency }\end{array}$ & $f$ & $\mathbf{k H z}$ & \multicolumn{2}{|c|}{38.0} \\
\hline Equipment & Pulse duration & $T$ & $\mathrm{~ms}$ & \multicolumn{2}{|c|}{1.00} \\
\hline
\end{tabular}


TABLE II. Ranges of parameter variations.

\begin{tabular}{cc}
\hline \hline Parameter & Range of variation \\
\hline$\rho_{1}$ & $\pm 1 \%$ \\
$c_{1}$ & $\pm 5 \%$ \\
$c_{2}$ & $\pm 3 \%$ \\
$\tau$ & {$[0,30]^{\circ} \mathrm{C}$} \\
$f$ & $\pm 0.5 \mathrm{kHz}$ \\
$T$ & $\pm 0.5 \mathrm{~ms}$ \\
$2 a$ & $\pm 0.1 \mathrm{~mm}$ \\
\hline \hline
\end{tabular}

variation is much larger than the manufacturing tolerance, but it is relatively small compared to the variation in the center frequency.
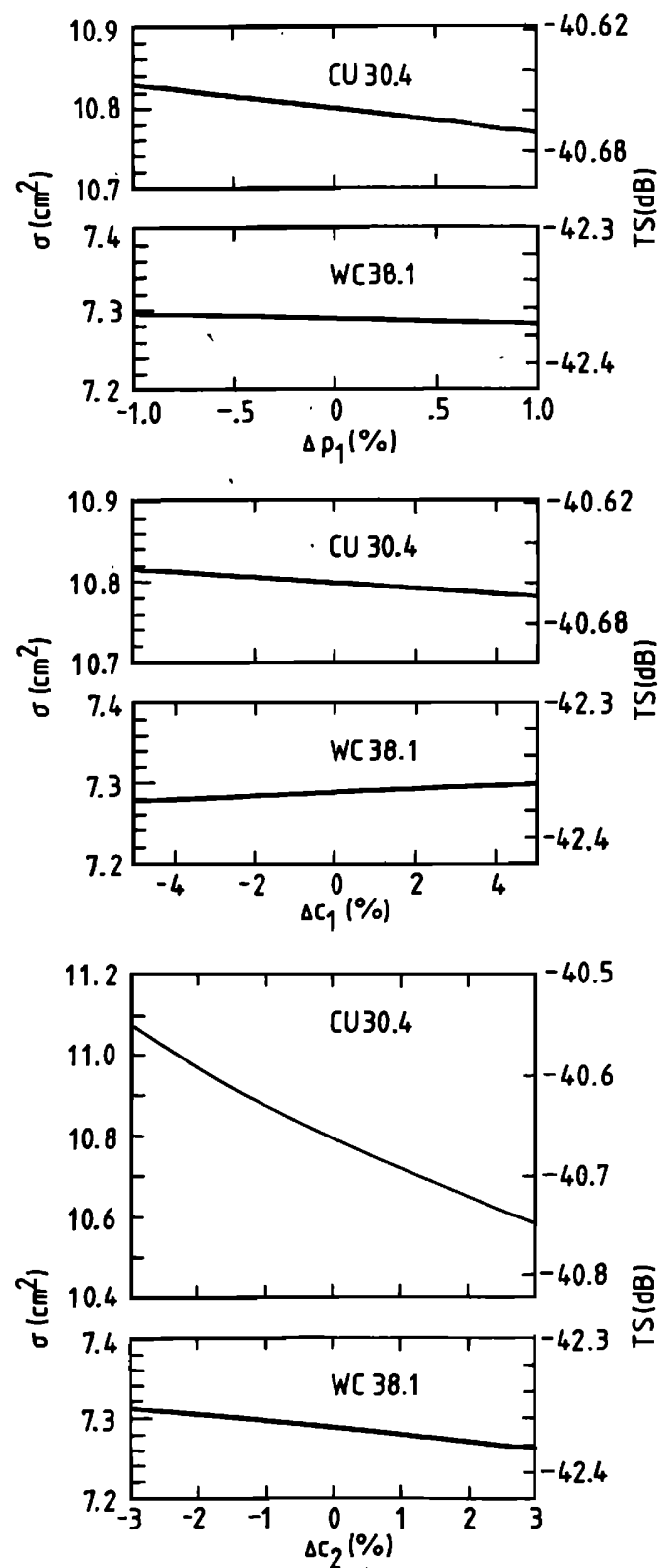

FIG. 1. Effects of material parameter variations on the backscattering cross sections $\sigma$ of two calibration spheres. $\rho_{1}$ is the density, $c_{1}$ the longitudinal sound speed, and $c_{2}$ the transverse sound speed. Nominal values of these and other parameters are given in Table I. TS denotes the target strength, defined as $10 \log \sigma /(4 \pi)$, where $\sigma$ is given in mks units.

\section{RESULTS AND DISCUSSION}

The effects of varying the material parameters from the nominal values are shown in Fig. 1. In each case, the effects for the tungsten carbide sphere are observed to be much less than the corresponding ones for the copper sphere. This is a consequence of tungsten carbide being much harder, in an acoustic sense, than copper at frequencies near $38 \mathrm{kHz}$. In no case are the variations large, although the examined parameter ranges are considerable. In the worst case, that of the copper sphere $\mathrm{Cu} 30.4$ when the transverse sound speed is varied, the maximum change from the nominal target strength is $\pm 0.1 \mathrm{~dB}$. Thus, even if the transverse sound speed were poorly known, use of the nominal target strength should allow a calibration to be performed with an accuracy of $\pm 0.1 \mathrm{~dB}$.

The temperature variation results in larger changes in the backscattering cross section. These are seen from Fig. 2 to be greater for the tungsten carbide sphere than for the copper sphere. Of course, the diameter of the copper sphere was calculated to minimize the temperature dependence. The diameter of the tungsten carbide sphere was merely selected from the limited range available in off-the-shelf items. Were the tungsten carbide sphere to be optimized in the manner of the copper sphere, then costly or nonstandard fabrication by machining or sintering would be necessary.

The precision of a marine calibration with the copper sphere will not be significantly affected by the weak temperature dependence of the target strength. The same is not true of the tungsten carbide sphere, for its target strength varies by $0.3 \mathrm{~dB}$ over the investigated temperature range. Since the temperature range from $0^{\circ}$ to $5^{\circ} \mathrm{C}$ contributes $0.2 \mathrm{~dB}$ to this variation, however, a single value of target strength can be found which will ensure a precision calibration over the range from $5^{\circ}$ to $30^{\circ} \mathrm{C}$. The temperature during a calibration

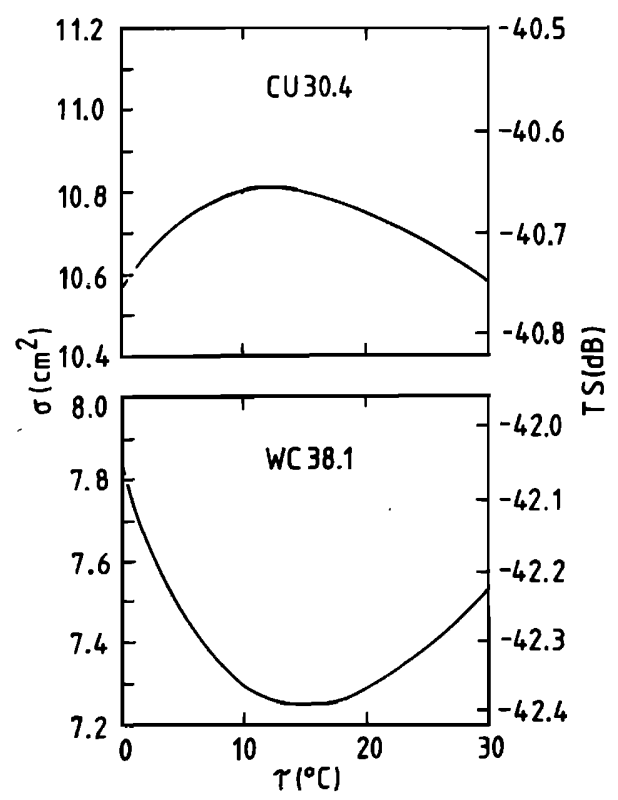

FIG. 2. Effects of temperature $(\tau)$ variation on the backscattering cross sections of two calibration spheres when they are immersed in sea water of nominal salinity 35 ppt. 
could be measured, of course, and the target strength value adjusted, if necessary.

Variations in the equipment parameters are seen from Fig. 3 to produce similar effects on the backscattering cross sections of both spheres. As in the case of the material parameters, the computed changes in target strength are small enough not to upset a precision calibration. The same conclusion applies to the diameter variations, whose effects, as noted above, are similar to those of frequency.

It is noted that the copper sphere is a stronger acoustic target than the physically larger tungsten carbide sphere. The nominal target strengths are -40.7 and $-42.4 \mathrm{~dB}$, respectively. This illustrates the greater flexibility afforded by group III materials like copper, compared to those of group II, such as tungsten carbide. In the case of the harder, group II materials, which might $a$ priori be considered more desirable for calibration applications, the range of variation
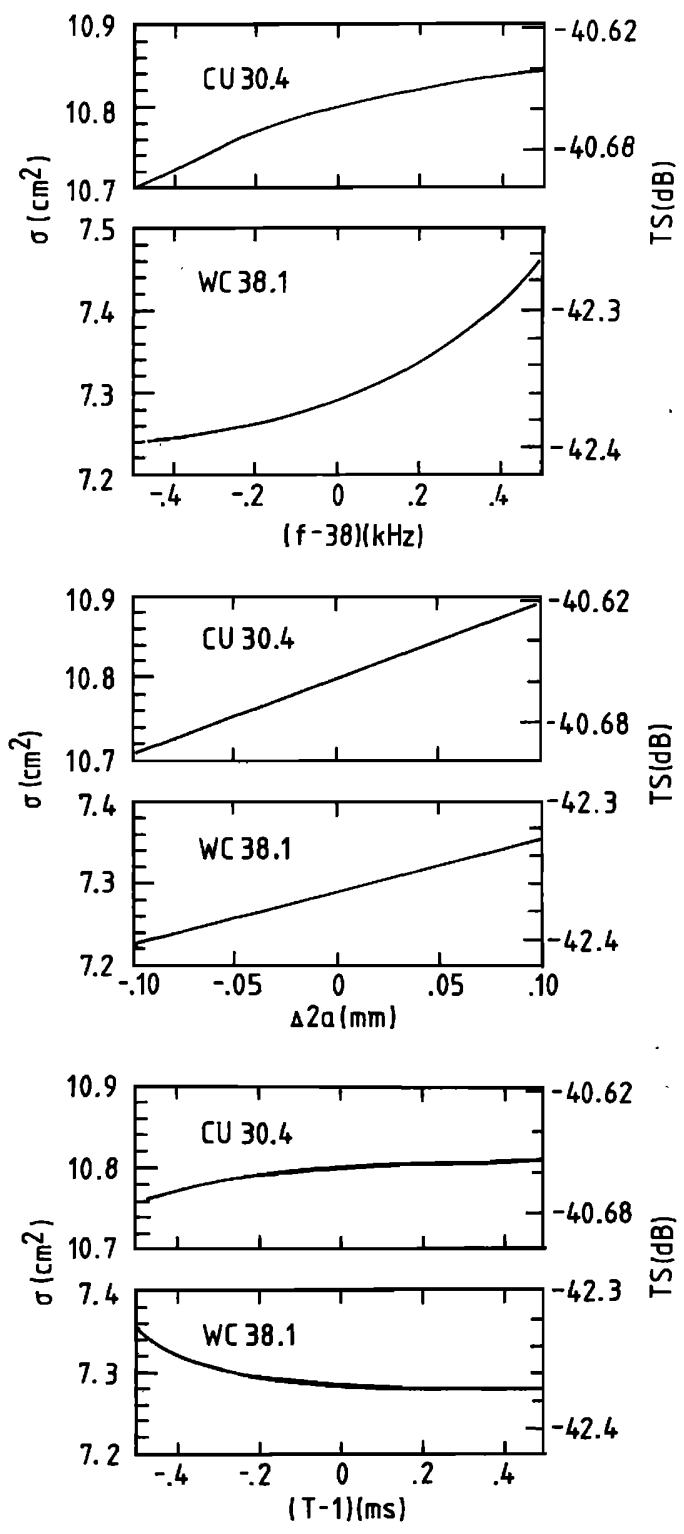

FIG. 3. Effects of variations in the equipment parameters and the diameter $2 a$ on the backscattering cross sections $\sigma$ of two calibration spheres. $f$ is the center frequency and $T$ is the duration of the transmit pulse. in backscattering cross section is smaller than that of materials in group III. The elasticity of these less hard materials may be exploited in a manner not possible with comparably sized specimens of harder materials.

\section{CONCLUSIONS}

The effects of variations in material and equipment parameters are generally small for both the copper and tungsten carbide spheres. The greater hardness of the tungsten carbide sphere results in the target strength having an extremely weak dependence on the material parameters. However, this confers no particular advantage with respect to variations in the equipment parameters, for which the two spheres show comparable effects.

Temperature has a greater effect than the other considered parameters, especially in the case of the tungsten carbide sphere below $5^{\circ} \mathrm{C}$. Insofar as the temperature can be measured or otherwise determined in a calibration exercise, corrections to the nominal target strength may be computed in order to preserve the $0.1-\mathrm{dB}$ precision.

Both copper and tungsten carbide are excellent materials for precision calibration spheres. Such considerations as cost, the need for a particular target strength, and acoustic robustness, among others, must dictate the choice between these in any particular application. It is evident that hardness is not the only, or even the most important, property to be considered in selecting a reference target for the calibration of acoustic systems.

'R. H. Wallace, H. V. Hillery, G. R. Barnard, B. M. Marks, and C. M. McKinney, "Experimental investigation of several passive sonar targets," J. Acoust. Soc. Am. 57, 862-869 (1975).

${ }^{2}$ L. R. Dragonette, S. K. Numrich, and L. J. Frank, "Calibration technique for acoustic scattering measurements," J. Acoust. Soc. Am. 69, 11861189 (1981).

${ }^{3}$ D. N. MacLennan, "Target strength measurements on metal spheres," Scottish Fisheries Research Report (25) (1982).

${ }^{4} K$. G. Foote, "Optimizing copper spheres for precision calibration of hydroacoustic equipment," J. Acoust. Soc. Am. 71, 742-747 (1982).

${ }^{5}$ K. G. Foote, "Constancy in target strength of optimal, elastic calibration spheres," in Proceedings of the IOA Underwater Acoustics Group Conference "Advances in Underwater Acoustics, Portland, England, 1-2 December 1981 (Institute of Acoustics, Edinburgh, 1981), pp. 11/1-6.

${ }^{6}$ K. G. Foote, "Maintaining precision calibrations with optimal copper spheres," J. Acoust. Soc. Am. 73, 1054-1063 (1983).

${ }^{7}$ K. G. Foote, H. P. Knudsen, G. Vestnes, R. Brede, and R. L. Nielsen, "Improved calibration of hydroacoustic equipment with copper spheres," Coun. Meet. Int. Coun. Explor. Sea 1981/B:20, Copenhagen, Denmark, $18 \mathrm{pp}$.

${ }^{8} \mathrm{~J}$. Zieniuk and R. C. Chivers, "Measurement of ultrasonic exposure with radiation force and thermal methods," Ultrason. 14, 161-172 (1976).

${ }^{9}$ F. Dunn, A. J. Averbuch, and W. D. O'Brien, Jr., “A primary method for the determination of ultrasonic intensity with the elastic sphere radiometer," Acustica 38, 58-61 (1977).

${ }^{10} \mathrm{~L}$. W. Anson and R. C. Chivers, "Frequency dependence of the acoustic radiation force function $(Y p)$ for spherical targets for a wide range of materials," J. Acoust. Soc. Am. 69, 1618-1623 (1981).

"C. A. Hampel, "Physical properties of metals," in Rare Metals Handbook (Reinhold, New York, 1961), 2nd ed., pp. 687-701.

${ }^{12}$ Handbook of Chemistry and Physics, edited by R. C. Weast, (The Chemical Rubber Company, Cleveland, 1968), 49th ed.

${ }^{13}$ R. C. Chivers and L. W. Anson, "Calculations of the backscattering and radiation force functions of spherical targets for use in ultrasonic beam assessment," Ultrason. 20, 25-34 (1981). 
${ }^{14} \mathrm{~T}$. Hasegawa and K. Yosioka, "Acoustic radiation force on fused silica spheres, and intensity determination," J. Acoust. Soc. Am. 58, 581-585 (1975).

${ }^{15}$ V. G. Welsby and J. E. Hudson, "Standard small targets for calibrating high frequency sonars," J. Sound Vib. 20, 399-406 (1972).

${ }^{16}$ Copper: The Science and the Metal, Its Alloys and Compounds, edited by A. Butts (Reinhold, New York, 1954).

${ }^{17}$ M. J. Druyvesteyn and J. L. Meyering, "Elastical constants in the system Cu-Zn," Physica (Utrecht) 8, 1059-1074 (1941).

${ }^{18} \mathrm{G}$. Faninger, "Die elastischen Konstanten von Kupfer-Nickel-Vielkristallen," Z. Metallkd. 60, 601-605 (1969).

${ }^{19}$ L. M. T. Hopkin, H. Pursey, and M. F. Markham, "Precise measurements of the elastic constants of copper and silver base alloys," $Z$. Metallkd. 61, 535-540 (1970).

${ }^{20} \mathrm{~L}$. S. Cain and J. F. Thomas, Jr., "Elastic constants of $\alpha$-phase Cu-Al alloys," Phys. Rev. B 4, 4245-4255 (1971).

${ }^{21}$ R. A. Wilkins and E. S. Bunn, Copper and Copper Base Alloys (McGrawHill, New York, 1943).

${ }^{22} \mathrm{~K}$. Iokibe and S. Sakai, "The effect of temperature on the modulus of rigidity, and on the viscosity of solid metals," Philos. Mag. 42, 397-418 (1921).

${ }^{23} \mathrm{~T}$. Kawai, "On the change of the modulus of rigidity in different metals caused by cold working," Tohuku Univ. Sci. Rep. 20, 681-709 (1931).
${ }^{24} \mathrm{~F}$. Birch, "The effect of pressure on the modulus of rigidity of several metals and glasses," J. Appl. Phys. 8, 129-133 (1937).

${ }^{25}$ J. Lobdell, G. F. Shinopulos, and D. N. Fillia, "An instrument to measure transverse strain," AVCO Corp. Res. Rep. RAD-TM-63-13 (1963) (NTIS Rep. No. AD 438571)

${ }^{26} \mathrm{H}$. H. Wawra, "Uber die Abhängigkeit des Elastizitäts-und Torsionsmoduls gezogener Kupfer-und $\alpha$-Messingstangen von der Anlasstemperatur," Z. Metallkd. 59, 268-272 (1968).

${ }^{27} \mathbf{K}$. G. Foote, "Refined determination of the rigidity modulus by echo sounder measurement,"' in 1981 Ultrason. Symp. Proc., Chicago, IL, 14 16 October 1981 (IEEE, New York, 1981), pp. 900-903.

${ }^{28}$ J. J. Faran, Jr., "Sound scattering by solid cylinders and spheres," J. Acoust. Soc. Am. 23, 405-418 (1951).

${ }^{29} \mathrm{D}$. Gerlich and G. C. Kennedy, "The elastic moduli and their pressure derivatives for tungsten carbide with different amounts of cobalt binder," J. Appl. Phys. 50, 3331-3333 (1979).

${ }^{30}$ J. R. Frederick, Ultrasonic Engineering (Wiley, New York, 1965).

${ }^{31}$ G. Dietrich, "Ozeanographie. Physikalische Eigenschaften des Meerwassers. Allgemeiner Teil," in Landolt-Börnstein Zahlenwerte und Funktionen (Springer-Verlag, Berlin, 1952), 6th ed., Vol. 3, p. 428.

${ }^{32} V$. A. Del Grosso, "New equation for the speed of sound in natural waters (with comparison to other equations)," J. Acoust. Soc. Am. 56, 1084-1091 (1974). 